\title{
„It’s ComforTable“: Ansätze zur Erforschung von Interaktionen an Tabletop-Displays im Ausstellungsraum
}

\section{Einleitung}

„Bitte nicht anfassen!“ Das Nicht-Berühren ist ein routinisierte Verhaltensweise, die zum Museumsbesuch ebenso dazu gehört, wie es dazu gehört, Ausstellungsräume perzipierend $\mathrm{zu}$ durchschreiten, d.h. Exponate betrachtend, erklärende Tafeln lesend, vielleicht dem Audioguide lauschend. Angefasst wird das Exponat selten. Ein Exponat, das angefasst und benutzt werden soll, muss also seinen besonderen Status vermitteln. Dabei stellen sich etliche Fragen: Wodurch wird ein Gegenstand zum Exponat? Wird er es schon dadurch, dass er sich in der räumlichen Umgebung eines Museums oder einer Ausstellung befindet? Wird er es nur dann, wenn er eine Nähe zum Thema der Ausstellung aufweist oder dadurch, dass ihn ein Schild als Exponat ausweist? Hat am Ende der Besucher eine Art Mitsprache- oder Mitdefinitionsrecht und wird all jenes zum Exponat, was vom Besucher betrachtet oder diskutiert wird? In der Regel entscheidet der Kurator einer Ausstellung darüber, welche Gegenstände als Exponate aufgenommen und ,gedacht' werden und welche nicht. Welche Lesart und Benutzung sich dem Besucher transportieren, ist jedoch zuletzt von dessen Blick auf das Objekt und der Bedeutungszuweisung abhängig. Beobachtbar wird die Bedeutungszuweisung anhand der Benutzung.

Was ein Objekt einem Besucher, der potenziell Betrachter oder Benutzer ist, mitteilt, ist eine Frage des Designs. Ueding (2008) verortet diese Frage im Bereich des Rhetorischen. Design nach heutiger Lesart habe eine „Wirkform“, die Form hat also eine sinnlich-intensive Wirkung (ebd.:82). Ist deren Herstellung ein bewusster Prozess, so ist damit auch eine Wirkungsintention verbunden, das Objekt 
soll einen Adressaten erreichen und ihn beeinflussen, ihn möglicherweise sogar zu etwas bewegen (ebd.:83, Mondada/Schmitt 2010:7ff.). Mit anderen Worten: Design hat eine persuasive Dimension. Genau wie sprachliches Handeln von Zielen bestimmt werde, so folge Design einem Zweck, einer Funktion. Gleichzeitig habe die Formensprache mehrere Möglichkeiten, um Ziele zu erreichen, weswegen es sich lohne, die unterschiedlichen Wirkrichtungen zu untersuchen (Ueding 2008:83). „Dieser Spielraum [unterschiedlicher Wirkrichtungen] gewinnt in dem Augenblick eine besondere Bedeutung, in dem ein Produkt gegen ein anderes antritt, gar mehrere Produkte miteinander um denselben Adressaten konkurrieren, in dem sich also, rhetorisch gesprochen, eine agonale Situation herstellt" (ebd.).

Die agonale Situation, um die es im vorliegenden Artikel geht, ist eine Ausstellung bzw. ein Museum, das interessierende Objekt ein Tabletop, der mit anderen Ausstellungsgegenständen konkurriert. Der Tabletop wurde unter dem Namen ComforTable im Rahmen des Graduiertenkollegs CrossWorlds (GRK 1780/1) an der Technischen Universität Chemnitz in Zusammenarbeit von Informatikern und Ethnografinnen entwickelt (Storz et al. 2015). Tabletop bezeichnet in Analogie zu den Bezeichnungen Desktop und Laptop die Position eines Computers oder Displays anstelle einer Tischplatte (Müller-Tomfelde/Fjeld 2010:2). Im Fall des ComforTable ist ein Display in die Tischplatte integriert, das gleichzeitig das Interface zum Computer zur Verfügung stellt (siehe Abb. 1 in Kap. 4). Realisiert ist das Interface als Multi-Touch Schnittstelle, d.h. die Aktionen werden durch Berührung (drücken, schieben, drehen) gesteuert. Weil mehrere Eingaben gleichzeitig möglich sind, ist ein solcher Tabletop geeignet für Mehr-Personen-Interaktionen (im Unterschied zu Single-Touch Displays, wie z.B. Tablets) (ebd.:13, Storz et al. 2015:115).

Der Tisch wurde für die Dauerausstellung im Sächsischen Industriemuseum Chemnitz entworfen und bietet Besuchern die Möglichkeit, ein Kartensortierspiel zu spielen, das in zwei Versionen vorhanden ist: In einem Set müssen Erfindungen, im zweiten Set Exponate der Ausstellung sortiert werden. Am Spiel können ein bis vier Spieler teilnehmen. Storz et al. beschreiben die Funktion des ComforTable wie folgt: „Most of all we wanted to create an artefact which prompts visitors to occupy themselves with contents of the exhibition but also with each other in a playful and pleasant manner" (2015:116f.). Mit diesem Designprogramm verfolgt der Tabletop die klare persuasive Absicht, Museumsbesucher miteinander in Kontakt zu bringen, oder, anders formuliert, in eine gemeinsame Interaktionssituation zu versetzen. Dabei befindet sich der Tisch als gestaltetes Objekt in einer räumlichen Umgebung mit anderen gestalteten Objekten. Die Erfassung und Analyse von Interaktionsereignissen kann daher die räumliche Umgebung nicht ignorieren. Der Tabletop als zentrales Element des Interaktionsereignisses muss auch als zentrales Element der räumlichen Umgebung begriffen werden.

In der direkten räumlichen Umgebung des Tabletops finden sich klassische Exponate des Industriemuseums. Auf der Abbildung in Kapitel 4 sind im Hin- 
tergrund z.B. Exemplare der Automarke Trabant zu sehen. Im nicht sichtbaren Umfeld gibt es außerdem eine Schnürsenkel-Maschine und eine Textilstraße. Im Kontext der Ausstellung konkurrieren also Objekte zur Betrachtung mit Objekten zur Benutzung. Die Forderung zeitgenössischen Produktdesigns - „form follows function“ - zielt darauf ab, dass die intendierte Benutzung am Objekt ,sichtbar“ wird (Norman 1989:24). Dieser Zusammenhang von Form und Funktion findet sich auch in üblichen Forschungsfragestellungen der Gesprächsanalyse. Diese fokussieren die Funktion, die eine bestimmte beschreibbare Form einer Äußerung erfüllt (z.B. Deppermann 2008:14f.).

Im Sinne eines Werkstattberichts möchte ich in diesem Artikel Überlegungen zur Erforschung von Mehr-Nutzer-Interaktionen am Tabletop und erste empirische Beobachtungen zur Interaktionsarchitektur vorstellen. Die Konzepte Interaktionsarchitektur und Interaktionsraum (Hausendorf/Schmitt 2013, 2014) bilden den konzeptionellen und methodischen Rahmen des vorliegenden Beitrags, der zum Ziel hat, Verbindungen zwischen Designforschung, Mensch-Maschine-Interaktion, Ausstellungskommunikation und Interaktionsforschung nachzuzeichnen. Zuerst wird der Ausstellungsraum Museum kurz beschrieben und die Konzepte Interaktionsarchitektur und Interaktionsraum werden vorgestellt (Kapitel 2), um anschließend ihre methodische Brauchbarkeit für die Analyse von Objektgestaltung und Interaktionsereignissen zu diskutieren (Kapitel 3). Kapitel 4 stellt erste Beobachtungen einer Interaktionsarchitekturanalyse des ComforTable - als Bericht einer Datensitzung - vor. Aus diesen Ergebnissen werden allgemeine Überlegungen für eine weitere analytische Vorgehensweise und konkrete Fragen für eine anschließende Interaktions(raum)analyse entwickelt (Kapitel 5). Abschließend wird die Anschlussfähigkeit der entwickelten Fragestellungen erwogen und zur Diskussion gestellt (Kapitel 6). ${ }^{1}$

\section{Konzepte: Interaktionsarchitektur und Interaktionsraum im Ausstellungszusammenhang}

\subsection{Ausstellungsraum und -kommunikation}

Der ComforTable wurde für ein Museum entworfen und damit für eine besondere Umgebung. Was Ueding (2008) für Objekte im Allgemeinen feststellt, gilt für Ausstellungsräume wie Museen und deren Bestandteile in besonderer Weise: In

${ }^{1}$ Die Arbeit an diesem Projekt wird finanziert von der Deutschen Forschungsgemeinschaft (DFG) unter der Fördernummer 1780/1 (Graduiertenkolleg CrossWorlds). Die Analyse wäre nicht zustande gekommen ohne das Kolloquium ,Sprache und Raum‘ (SpuR) an der Universität Zürich unter der Leitung von Heiko Hausendorf und Reinhold Schmitt. Bei ihnen und allen Teilnehmern des Kolloquiums möchte ich mich für die wertvollen Impulse, Anmerkungen und kritischen Fragen sehr herzlich bedanken. Den Herausgebern der Zeitschrift danke ich sehr für ihre präzisen und äußerst hilfreichen Anmerkungen zu einer früheren Version des Artikels. 
aller Regel folgen Ausstattung und Gestaltung museumspädagogischen Erwägungen. Im Fall des Tabletops heißt das, der Besucher soll zu dessen Benutzung bewegt werden. Der Besucher ist in diese Planung als Adressat bereits einbezogen. Das entspricht dem Gedanken von Hausendorf (2012:140), in der Gestaltung benutzbarer Dinge stecke auch recipient design, insofern als dass ihre Benutzbarkeit bereits in den Entstehungsprozess eingeflossen sei. So bezeichnet Kesselheim (2012:189) die Museumsausstellung auch als Umgebung, ,die besonders geeignet ist, die Rolle der materiellen Umwelt für die in ihr stattfindende Interaktion auszuloten".

Pitsch (2012:233) argumentiert in eine ähnliche Richtung und hebt Interaktion im Museum als Untersuchungsgegenstand hervor, weil Ausstellungsräume „eine bedeutungsvolle materielle Umwelt [anbieten], in der Objekte und Artefakte zum Zweck des Betrachtet-Werdens ausgestellt sind und in der Regel durch spezifische Formen der Präsentation als ,wertvoll' ausgewiesen werden“. Darüber hinaus müssten sich Besucher zum Betrachten und Manipulieren von Objekten im Raum bewegen (ebd.). Pitsch zeigt, und das ist für die vorliegende Untersuchung besonders interessant, dass der Status von Objekten für Besucher nicht per se eindeutig ist. In ihrer Analyse zeigt sich, dass ein Objekt je nach Zeitpunkt und thematischer Relevant-Setzung im Verlauf einer Ausstellungsführung seine Bedeutung verändern kann (ebd.:239ff.). Welche Bedeutung Besucher einem Objekt zuweisen, zeigen sie durch ihre Benutzung.

Hausendorf und Kesselheim (2007) betonen die Besonderheit von Ausstellungskommunikation. Diese ergibt sich daraus, dass Planung und Gestaltung des Ausstellungsraums zeitlich vor dessen Benutzung durch die Besucher liegen. Um diese zwei Zeitpunkte im kommunikativen Prozess zu berücksichtigen, müsse die Erschaffung des kommunikativen Produkts getrennt von dessen Perzeption und Benutzung betrachtet werden. In den Konzepten von Interaktionsarchitektur und Interaktionsraum ist diese getrennte Betrachtung bereits angelegt, wie im Folgenden deutlich werden soll. Im Begriff Ausstellungskommunikation wird der Gestaltungsprozess als Teil der Kommunikation mit dem Besucher verstanden. Die in der Ausstellung stattfindende Interaktion der Besucher wird als „Spezialfall“, oder eine mögliche Erscheinungsform, von Kommunikation konzeptualisiert (ebd.:339). Folglich müsse die Analyse des gestalteten Ausstellungsraums als kommunikative Erscheinungsform von der Analyse tatsächlicher Interaktion der Besucher in der Ausstellung methodisch getrennt werden (ebd.:339ff.).

\subsection{Interaktionsarchitektur}

Der hier verwendete Begriff von Interaktionsarchitektur folgt dem Vorschlag von Hausendorf und Schmitt (2013:3) und ,steht für die Frage, wie die Architektur von Räumen Interaktion (wenn auch nicht determinieren und verhindern, so 
doch) ermöglichen und nahelegen kann und wie man diese interaktionsarchitektonischen Implikationen empirisch rekonstruieren kann. Unter ,Architektur' verstehen wir dabei heuristisch all das, was vom gebauten Raum (aus Stein, Beton, Holz, ...) über den gestalteten Raum (,Innenarchitektur') bis zum ausgestatteten Raum (z.B. Technik, Dekoration) reicht“.

Der Tabletop im Ausstellungsraum hat in dreierlei Hinsicht interaktionsarchitektonische Implikationen. Zunächst befindet er sich in einem umbauten Raum - einer sanierten ehemaligen Gießerei, die heute das Sächsische Industriemuseum Chemnitz beherbergt. Die Hallen der Gießerei sind auf spezifische Weise gestaltet, die der Nutzung als Ausstellungsfläche gerecht werden muss. Dazu gehören Vitrinen, Schränke, Podeste u.ä. Schließlich ist der Raum ausgestattet und dekoriert: Er ist farblich gestaltet, es gibt technische Geräte, wie den ComforTable oder spezifisch ausgerichtetes Licht. Die Gesamtheit dieser Merkmale, die Innenarchitektur, Flächen, Begrenzungen und Nutzungspotenziale sowie Orientierungen, sind „Kandidaten“ für räumliche Aspekte der Interaktion (Schmitt 2013:47). Folglich greift „eine ausschließlich auf die Rekonstruktion der körperlich-räumlichen Konstellationen abzielende Analyse, die das Potenzial und die Beschränkungen der physikalischen Umgebung als wichtige konstitutive Größe ignoriert, [...] zu kurz" (Schmitt 2013:51).

Das Konzept der Interaktionsarchitektur liefert einen Rahmen für die Beschreibung des Tabletops als designtes Objekt mit einer persuasiven Absicht in einer spezifischen räumlichen Umgebung. Es geht darum, die Potenziale des Raumelements für die initiierten Interaktionsereignisse auszuloten. Dieser Blick auf die Gegebenheiten eines Raums respektive eines Objekts lässt die Interaktionsereignisse für die weitere Analyse umso interessanter werden und führt zu der Frage: Was könnte hier passieren?

\subsection{Interaktionsraum}

Traditionell spielt der Raum in der Linguistik vor allem als Verweisraum eine Rolle. In diese Richtung gehen Beobachtungen zur Funktion des Raums als Raumreferenz, bei der räumliche Orientierungen als Referenzrahmen in Beschreibungen etabliert werden (weiterführend dazu Stutterheim/Kohlmann 2001, Fricke 2007, Stukenbrock 2015).

Für die Soziologie zeigt Schroer (2006) eine Vielzahl von Ansätzen zur Konzeptualisierung von Raum, formuliert jedoch als Desiderat das Fehlen einer konsequenten „Raumsoziologie“. Als wiederkehrenden Ansatz zeichnet Schroer ein Verständnis von Raum nach als etwas, das auf soziale Operationen zurückzuführen ist (ebd.:28). Raum kann demnach nur da entstehen, wo wir sind und durch das, was wir (miteinander) tun. Dieser Grundgedanke ist zentral für das Konzept Interaktionsraum. „Interaktionsraum bezeichnet die räumlichen Konfigurationen, die 
die Interaktanten im Verlauf ihrer Aktivitäten herstellen“ (Mondada 2007:55). Mit diesem Konzept wird Raum in der Interaktionalen Linguistik als Ressource für die Interaktion, die in ihm stattfindet, begriffen. Dies auch, weil mit der Erfassbarkeit von Interaktionsereignissen im Alltag durch Videoaufnahmen die Beschreibung von Raum und die Analyse von Interaktion im Raum zwangsläufig relevant wurde. Zuvor war es in der Konversationsanalyse eher üblich, den Raum und dessen Gegebenheiten als ethnografisches Detail zu behandeln. Seine Wirkung auf die Interaktion und auch seine Bearbeitung in der Interaktion waren nicht Teil der Analyse und der Zusammenhang zwischen räumlichen Gegebenheiten und den Aktivitäten, die in Bezug auf diese stattfinden, blieb weitgehend unberücksichtigt (Mondada 2007). Mit dem Einzug der Videotechnik wurde die Konversationsanalyse zunehmend um multimodale Fragestellungen erweitert: Der Begriff multimodal verweist in diesem Zusammenhang „sowohl auf Gesten, Blicke, Mimik, Körperbewegung und -haltung, Positionierung und Bewegung im Raum als auch auf die Benutzung von Gegenständen“ (Gülich/Mondada 2008:115). Folgerichtig haben sich Konversationsanalyse und Interaktionale Linguistik programmatisch und analytisch in den letzten Jahren gewinnbringend mit Phänomenen des Raums beschäftigt (z. B. Schmitt 2007, 2013, Hausendorf/Schmitt 2013, 2014, Hausendorf et al. 2012). Das Konzept Interaktionsraum also „lenkt die Aufmerksamkeit auf die räumlichen Arrangements der Körper der Interaktanten und ihre wechselseitige Ausrichtung und damit auf die Verfahren, mit denen sie sich im Hinblick auf ihr gemeinsames Handeln im Raum koordinieren“ (Mondada 2007:55). Mondada plädiert dafür, den praxeologischen Ansatz einer Raumkonzeption zu vertiefen: „Er muss sich explizit mit den Beschränkungen durch den Raum sowie mit dessen Rolle als Ressource für die Interaktion befassen und so seine Verbindungen zum Handeln aufzeigen, [...] er muss die Materialität und die Formbarkeit des Raums berücksichtigen, welcher sowohl der Interaktion äußerlich ist als auch von ihr gestaltet wird“ (ebd.:62). In dieser Konzeption ist der Raum nicht einfach vorhanden. Vielmehr verändert er sich im Laufe der Handlung durch die Handlung selbst; Handlung und Raum sind miteinander verbunden und aufeinander bezogen: „Der Interaktionsraum ist weder vom umgebenden Raum vorgegeben noch ein für alle Mal festgelegt. Er ist die praktische Hervorbringung der Interaktanten, eine Konfiguration, die im gemeinsamen Handeln emergiert und auf dessen praktische Zwecke zugeschnitten ist" (ebd.:87).

Die Konzepte Interaktionsarchitektur und Interaktionsraum behandeln zwei verschiedene, aber aufeinander bezogene Betrachtungsweisen von Raum. Die Interaktionsarchitektur konzeptualisiert den gegebenen Raum und die Möglichkeiten, die dieser für Interaktionen bietet. Im Konzept des Interaktionsraums geht all das auf, was interaktional im und durch Raum stattfindet. Mit dem Interaktionsraum werden die Manipulationen und Nutzungsweisen der Teilnehmer im betrachteten Raum beschrieben. Interaktanten können also auf gebauten, gestalteten und ausgestatteten Raum als Ressource zurückgreifen, um Interaktionsraum herzustellen. 


\section{Vorgehensweise}

In Kapitel 2 sollte die enge Verzahnung der Konzepte Interaktionsarchitektur und Interaktionsraum dargestellt und gezeigt werden, dass auch die Ausstattung des Raums bzw. seine Beschaffenheit methodisch in die Analyse einbezogen werden muss. Die Gegebenheiten werden nicht nur durch Interaktion verändert oder geformt, sie wirken auch strukturierend und schaffen Benutzungsmöglichkeiten. So kann ein Tisch dazu benutzt werden, Dokumente auszubreiten, etwas abzustellen oder sich unter Zuhilfenahme eines anderen verfügbaren Möbels daran zu setzen. Genauso gut kann er aber von einem Kind zum Haus oder zu einer Höhle erklärt und, unter die Tischplatte kriechend, als solche(s) benutzt werden.

Der vorliegende Artikel orientiert sich an der von Hausendorf und Schmitt (2014) vorgeschlagenen strikten Trennung von Architekturanalyse und Interaktionsanalyse. Dokumente für ersteres sind Fotografien des Raums bzw. der räumlichen Gegebenheiten, für letzteres werden Standstills, Audio, Transkript, Video benutzt. „Nur so lässt sich der jeweilige Eigenwert der Analysen und der mit ihnen verbundenen Dokumente auch zur Geltung bringen" (Hausendorf/Schmitt 2014:5). Aus diesem Grund beschreibe ich in diesem Beitrag zunächst den ersten Schritt (Architekturanalyse) und verweise auf die zukünftig durchzuführende Interaktionsanalyse.

Das methodische Prinzip der geteilten Analyse lautet: Im Rahmen der Architekturanalyse ist aus der spezifischen Struktur so viel wie möglich herauszuarbeiten. Dies wird erreicht, wenn sich die Analyse zunächst ausschließlich auf Fotografien bzw. Bilder der räumlichen Gegebenheiten bezieht (ebd.). Da es bei der Interaktionsarchitekturanalyse um die Analyse des Raums ohne die darin stattfindende zwischenmenschliche Interaktion geht, erscheint diese Herangehensweise schlüssig. Sie erleichtert zudem die visuelle Präsentation der Analyse (s.u. Kap. 4). Aus meiner Sicht gilt es darüber hinaus zu bedenken, dass der Raum beim Betrachter, der körperlich im Raum steht, einen spezifischen Eindruck macht. Dieser kann im Detail von dem Eindruck durch Fotografien abweichen, schon allein dadurch, dass das Foto nur einen Ausschnitt zeigen kann, an dessen Rand der Betrachter im Raum aber andere Elemente wahrnehmen kann. Auch der Fokus ist festgelegt - ich erfahre nicht ohne Weiteres etwas darüber, ob die fotografierten Objekte oder Elemente tatsächlich jene sind, die beim Betreten des Raumes als erstes ins Auge fallen. Ein zusätzlicher Einbezug ethnografischer Notizen zur Umgebung könnte helfen, den räumlichen Eindruck anzureichern. Diese Art der Daten ist um einiges subjektiver als Bilddokumente, da sie in der Regel nur von einer Person, zum Beispiel der erhebenden Forscherin, angelegt werden. Nicht zuletzt deshalb erweist sich bereits in dieser Phase der Analyse das Prinzip der Datensitzung als hilfreich. 
In der qualitativen Sozialforschung, zu der auch die Gesprächsanalyse gehört (Deppermann 2008:10), ist die Form der Datensitzung im Sinne eines ,peer debriefings“ eine übliche Form zur Qualitätssicherung und Validierung von Analyseergebnissen (Flick 2005:198). Sie kann beschrieben werden als „regelmäBige Besprechung mit anderen Forschern zur Aufdeckung blinder Flecke sowie Überprüfung der Angemessenheit von Interpretationen [...]“" (ebd.). Neben dieser Validierung von Interpretationen wird so auch „die Transparenz des Forschungsprozesses zum einen durch die öffentliche Diskussion vor der wissenschaftlichen community gesichert und zum anderen dadurch, dass in Datensitzungen prinzipiell der gesamte Forschungsprozess aufgegriffen werden kann" (Schwarze 2014:165, kursiv i.O.). Die Datensitzung ist damit u.a. ein wichtiges Mittel zur Intersubjektivierung und kann in verschiedenen Stadien des Forschungsprozesses wichtige Erkenntnisse zu dessen weiterer Gestaltung liefern. Gerade in Gruppen, die mit diesem Prinzip vertraut sind, entsteht so eine "Seh- und Deutungsgemeinschaft“ (Tuma 2013:67), deren Deutungen „das Ergebnis fein abgestimmter Rekonstruktionsarbeit (sind)" (ebd.:68). Tuma formuliert diese Beschreibung für die gemeinschaftliche Videoanalyse. Für die Betrachtung von Bildern zum Zweck einer Interaktionsarchitekturanalyse, wie im Folgenden, kann dies jedoch genauso gelten.

Schwarze (2014:171) betont, dass der Erfolg einer Datensitzung wesentlich von einer klaren Zielsetzung abhängig ist. Die Sitzung, deren Ergebnisse in Kapitel 4 dargestellt werden sollen, fand am 14. Oktober 2015 in Zürich als Veranstaltung des Kolloquiums, Sprache und Raum' statt. Sie verfolgte vor allem zwei Ziele:

- Sie sollte als Einstieg in die Interaktionsarchitekturanalyse fungieren und im Sinne des methodischen Prinzips, so viel wie möglich herauszuarbeiten (s.o.), möglichst viele Interpretationen und Perspektiven zusammentragen (Schwarze 2014:171).

- Sie sollte Fragen für die Interaktionsanalyse identifizieren, die im Forschungsprogramm einer Interaktionsraumforschung dann den nächsten Schritt darstellt.

Die Ergebnisse der Datensitzung repräsentieren den methodisch ersten Schritt im Programm der Interaktionsraumforschung, wie sie in Kapitel 2 dargestellt wurde. In dieser radikalen Trennung und Nacheinander-Betrachtung von Interaktionsarchitektur und Interaktionsraum, wie Hausendorf und Schmitt (2014) sie vorschlagen, ist es möglich, die Potenziale geplanter Räume zu beschreiben und deren Ausschöpfung, Nichtausschöpfung oder Umdeutung deutlich zu machen. In der Analyse der tatsächlich stattfindenden Interaktionsereignisse wird sich dann zeigen, wie konkrete Designelemente , funktionieren', in welcher Weise sie ihren Adressaten ansprechen und von sich überzeugen (oder auch nicht). Im Folgenden 
werden die Beschreibungen aus der Interaktionsarchitekturanalyse, wie sie in der Datensitzung gesammelt wurden, dargestellt und daraus Fragen für eine zukünftige Interaktionsanalyse entwickelt.

\section{Erste Analysen zur Interaktionsarchitektur}

Im Folgenden möchte ich zeigen, welche Benutzbarkeitshinweise des ComforTables verstehbar sein können. Benutzbarkeitshinweise werden gefasst als „Anknüpfungspunkte für Wahrnehmung, Bewegung und Handlung [...]. Im Alltag werden sie häufig wie selbstverständlich verstanden und körperlich ,beantwortet"“ (Hausendorf 2012:139). Diese unbewusst verständlichen Benutzbarkeitshinweise sind die Grundlage intuitiven oder ,menschenfreundlichen“ Designs, das darin besteht, Benutzung „,sichtbar“" zu machen (Norman 1989, 2004).

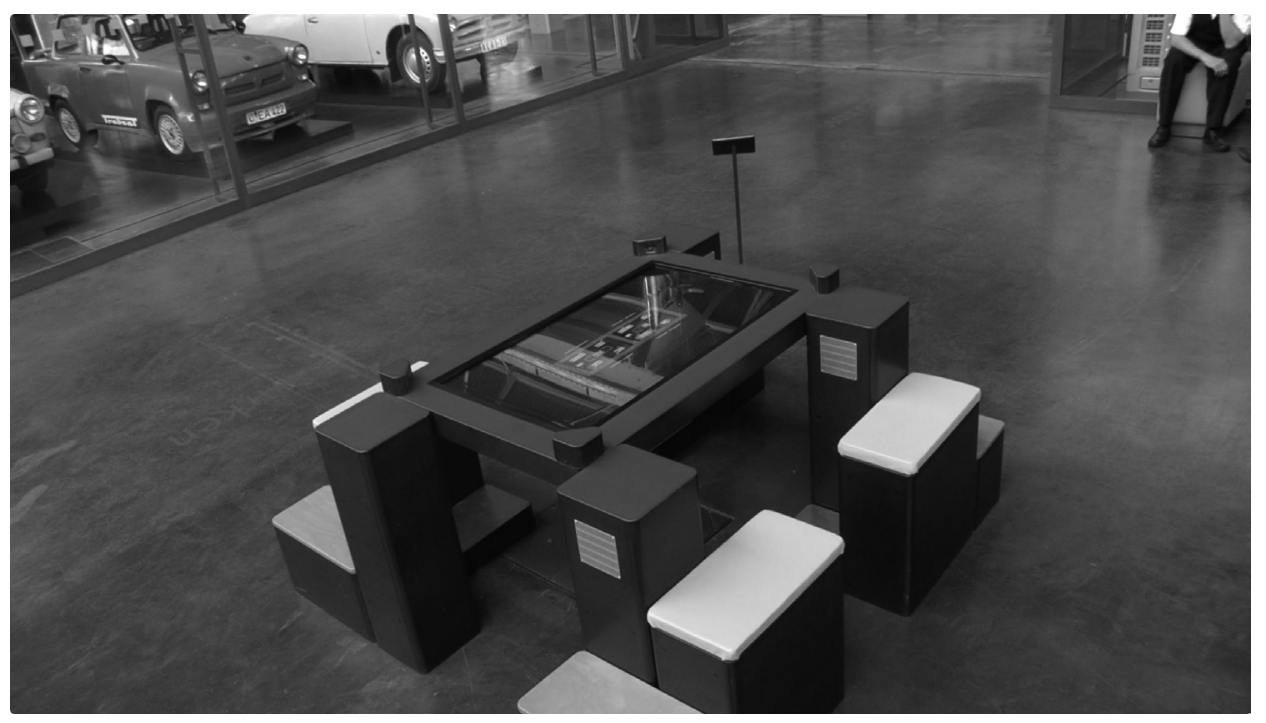

Abb.: ComforTable ${ }^{2}$

Der von Schmitt und Hausendorf (2013) vorgeschlagenen Analyse-Chronologie folgend, stand in der Datensitzung die Analyse der Interaktionsarchitektur im Vordergrund. Die Grundlage dafür bildet ein Still der Videoaufnahme (Abb.). Der besondere Wert dieser Vorgehensweise liegt darin, einen unverfälschten Blick

2 Der Videostill ist mit freundlicher Genehmigung entnommen aus Aufnahmen, die im Rahmen des Projekts „Crossworlds - Connecting virtual and real social worlds“ (DFG Graduiertenkolleg 1780/1) von Kalja Kanellopoulos und Michael Storz im Sächsischen Industriemuseum Chemnitz im Mai/Juni 2014 aufgezeichnet wurden (www.crossworlds.info). 
auf den Tabletop einzufangen, der im Prinzip dem Blick der Museumsbesucher entspricht oder diesem sehr ähnlich ist. Da die Datensitzungsgruppe aus Studierenden und Interaktionsforscherinnen nichts über den Designprozess des Tisches und über die Ausstellungskonzeption weiß, ist der Blick auf das Datum der Perspektive der vorübergehenden Museumsbesucher ähnlich.

Die Teilnehmer der Datensitzung erhielten zum Einstieg eine kurze Einführung zum Projekt, zum Gegenstand des Tabletops und einige wenige Informationen zum Standort des Geräts und seiner Einbettung in die Dauerausstellung des Museums. Der Zugang zum Bild erfolgte ,unsortiert' und ausschließlich orientiert an augenfälligen Merkmalen und Besonderheiten des Geräts. Charakteristisch für diese Herangehensweise war das Aufwerfen von Fragen, wie dieses und jenes Gestaltungselement gemeint sein könne. Verläuft die Sammlung solcher Fragen und Bemerkungen naturgemäß eher kumulativ, erscheint es mir nach der Auswertung sinnvoll, die Ergebnisse in sechs Element-Gruppen einzuteilen, die sich in der Sitzung ergeben haben: (1) Tisch/Gesamteindruck, (2) Sitze/Sitzgelegenheit, (3) Tischbeine, (4) Benutzung, (5) Oberflächen, (6) Display. Diese Ordnung ist nicht bewusst im Vorhinein zusammengestellt, sie ergibt sich ausschließlich aus der gemeinsamen Sammlung in der Datensitzung und einer nachträglichen Kategorisierung. Ich möchte insofern auch nicht ausschließen, dass Elemente fehlen oder vernachlässigt erscheinen könnten. Im Austausch über die Beobachtungen sind Fragen formuliert worden, die die Grundlage für die zukünftige Interaktionsanalyse bilden sollen. Diese Fragen werden hier jeweils im Fettdruck dargestellt und am Ende des Kapitels noch einmal zusammengefasst.

\section{(1) Tisch/Gesamteindruck}

Die Hauptfunktionalität des ComforTable besteht in einem digitalen Kartenspiel, woraus sich ein Spannungsverhältnis zwischen sichtbarer Interaktionsarchitektur und Sozialtopografie ergibt. Während letztere Ausstellungsstücke für ein Museum per se vorsieht, schließt sie Spielgeräte zwar nicht aus, hält sie aber auch nicht unbedingt bereit. Die Architektur des Objekts wurde von der Analysegruppe mehrfach als widersprüchlich und irritierend bezeichnet. Methodisch vielleicht nicht ganz unproblematisch ist als Gegenstand der Analyse von vornherein der Tisch eingeführt und als solcher benannt worden. Die Situation der Museumsbesucher ist insofern jedoch ähnlich, als dass auf dem im Bild zu sehenden Schild ebenfalls die Bezeichnung Multi-Touch Tisch auftaucht und zumindest von einer Annäherungsseite aus auch wahrgenommen werden kann. Vor diesem Hintergrund ist die Feststellung eines Beobachters einzuordnen, dass das Objekt auf den ersten Blick wenig mit einem Tisch zu tun habe. Gleichwohl gibt es Elemente der Architektur, die ganz klar auf einen Tisch hindeuten - es gibt vier senkrechte Körper (Beine) und einen waagerechten, 
ebenen Körper (Platte). Auf dem Bild nicht eindeutig zu erkennen, am tatsächlichen Objekt aber durchaus, ist die Höhe des waagerechten Körpers ungefähr auf Hüftniveau, was ebenfalls als tischartig erkannt werden dürfte. Eine Einordnung als Tisch, so sie also erfolgt, ändert nichts an der globalen Feststellung, dass die Implikation des Objekts im ersten Moment nicht eindeutig ist. Daraus ergibt sich für die Interaktionsraumanalyse die grundsätzliche Frage: Wie wird das Objekt durch Besucher interpretiert bzw. konzeptualisiert? Für die Benutzung maßgeblich ist ein Hinweis auf die richtige Positionierung am Tisch, stehend oder sitzend, worauf weiter unten noch eingegangen werden soll. Im Ersteindruck bleibt festzuhalten, dass weder Display noch Anordnung der Körper ganz klar zeigen können, welche Körperhaltung sich für die Bedienung am besten eignet. Einen Hinweis auf die Seite oder Richtung der Annäherung gibt das Display, das eine der beiden langen Seiten nahelegt, da die Schrift sonst nicht lesbar ist (ethnografisches Wissen). Auch könnte man annehmen, dass ein Tisch als Gegenstand so etwas wie Sitzen voraussetzt. Gleichzeitig gibt es natürlich Tische, die als Stehtische eindeutig zu erkennen sind (Hausendorf/Schmitt 2014:9). Neben dem Tisch wurde von der Datensitzungsgruppe die Anleihe bei fest verschraubten Schulbänken ins Spiel gebracht und damit ein infantilisierender Charakter der Gesamtarchitektur. Ein Perspektivenwechsel auf Kinder als ,kleine Benutzer ${ }^{6}$ legt allerdings nahe, dass aus deren Perspektive etwas ganz anderes gesehen wird. So bleibt kleinen Kindern in der Seitenansicht des Tisches der rote Rahmen auf der Oberseite verborgen wie insgesamt das ganze Display. Das Objekt dürfte dann wie ein schwarzer Tunnel oder eine Brücke wirken, wie etwas also, das man um- und unterqueren, eventuell auch beklettern könnte. Völlig im Gegensatz dazu steht die Assoziation mit einer Kunstausstellung und einer Aufforderung durch das liegende Display, es von jeder der Zugangsseiten zu betrachten. Wie Nutzer das Problem der Aufenthaltsform am Display lösen, wird erst die Interaktionsanalyse zeigen.

\section{(2) Sitze/Sitzgelegenheit}

Die Grundform des ,Geräts ‘ weist Elemente eines Tisches auf: Es gibt vier Beine und eine große Platte, die die größte Fläche der Gesamtkonstruktion ausmacht. Daneben fallen sofort die vier farblich abgehobenen (gelb) und gepolsterten Flächen ins Auge, die als Sitzgelegenheiten verstehbar sind. Sonderlich bequem wirkten die Sitzflächen auf die Betrachterinnen nicht - eher so, als solle man nur eine begrenzte Zeit dort Platz nehmen. Eine Information zum Wie des Sitzens halten die Flächen nicht bereit. Vielmehr stellt sich die Frage, wie man als Benutzer eigentlich sitzen soll. In Frage kommt die Variante mit Blick und Händen zum Display. Diese wird zumindest von dessen Position nahegelegt. Da die Sitzflächen jedoch über den effektiven Platz ,am Tisch' hinausgehen, werden zusätzliche Positionen denk- und verstehbar: Erstens mit dem Rücken zur Säule, denn diese hat mit der Sitzfläche zusammen die typische Form eines Stuhls mit Lehne. Zweitens seitlich - dann könnte die Säule als Armlehne 
fungieren, ebenfalls mit Blick vom Display weg. Es bleibt vorerst festzuhalten: Zwar sind die Sitze als solche zu identifizieren. Ihre Anordnung jedoch ist uneindeutig oder, anders formuliert, die Benutzungshinweise zum Sitzen sind mehrdeutig. Das Gleiche trifft auf die ebenfalls zu erkennenden niedrigeren Flächen zu, die nicht nur in der Höhe, sondern auch in der Farbe von den hellgelb gepolsterten Flächen abgesetzt sind. Es stellt sich zum Beispiel die Frage, ob eine dieser Flächen zum Abstellen der Füße gedacht ist oder die unterschiedlichen Höhen Sitzflächen für unterschiedlich große Besucherinnen zur Verfügung stellen sollen. Fragwürdig erscheint in diesem Zusammenhang die Anordnung der Flächen. Bei jener Ecke, die auf der Abbildung oben im Vordergrund ist, ,verbaut' das Tischbein den Zugang zum Display, wenn man, in welcher Weise auch immer, auf der bräunlichen Fläche Platz nimmt. An der im Bild linken Ecke ergibt sich ein ähnliches Problem. Das Sitzen dort ist nur möglich mit Blick auf die Autos oder in den Gang. In beiden Fällen kann das Display nicht erreicht werden. Von den grauen Flächen aus Blech, die jeweils vor den Sitzen erkennbar sind, wird eine andere Haltung nahegelegt, das Stehen. Das Ensemble gibt also irritierende Informationen, überlässt damit dem Besucher aber auch die Freiheit, alles Erdenkliche aus dem Angebot zu machen. Darüber hinaus könnten verschiedene Besuchergruppen angesprochen werden: Erwachsene mit den gelb gepolsterten Flächen, Kinder mit den niedrigeren, und damit einfacher zu erreichenden, braunen.

Für die Interaktionsanalyse ergeben sich daraus zwei Fragen. 1. (Wie) werden Deutungsprobleme durch Teilnehmerhandlungen offenbar? 2. Gibt es nutzergruppenabhängige Arten der Nutzung des Geräts? Die Sitzgelegenheiten beeinflussen eine weitere Dimension - jene von Nähe und Distanz. In der sichtbaren Anordnung bleibt zwischen den Ecken des Tisches nicht viel Raum, noch deutlich weniger bleibt, wenn alle als Sitzpositionen deutbaren Flächen belegt sind. Ein optischer Effekt der Sitze ist folglich eine Beschränkung des zur Verfügung stehenden Raumes am Tisch bzw. am Display. Eine Implikation dieser Verknappung des Raumes wäre, dass die Benutzung mit Freunden und Familie näher gelegt wird als die mit Fremden. Für die spätere Analyse der Interaktionen stellt sich damit die Frage: Was wird durch die räumliche Begrenzung ermöglicht beziehungsweise verhindert? $\mathrm{Zu}$ den ermöglichten Aktionen kann zum Beispiel das Zuschauen zählen. Raum dafür ergibt sich sowohl an den beiden Stirnseiten als auch auf den Sitzflächen, die breiter sind als normalerweise nötig. Gleichzeitig ergibt sich ein Verteilungs-Problem, denn augenscheinlich können zwar zwei Personen auf einem Polster sitzen, nicht aber gleichzeitig Zugang zum Display haben. Dies wird durch die Tischbeine verhindert.

\section{(3) Tischbeine}

Die Formgebung der Tischbeine hat sich in der Datensitzung als bemerkenswert herausgestellt. Ihre Implikationen sind widersprüchlich. Auf der einen Seite ge- 
hören Tischbeine, nicht selten deren vier, zum Konzept Tisch, was sie erwartbar und keineswegs besonders macht. Auf der anderen Seite fallen sie auf. So sind die Tischbeine des Tabletops nicht nur versetzt und neben statt unter der Tischplatte angebracht, jedes ist darüber hinaus auf eine andere Weise versetzt, so dass die Beine eher als ,Türme' erscheinen. Wie oben bereits beschrieben, verringern mindestens zwei der Tischbeine, nämlich jene, die im Vordergrund zu sehen sind, den Zugang zum Display. Beides ist eher ungewöhnlich und verstärkt die Frage nach ihrer Funktion. Sie sind offenbar nicht nur zur Stütze der Tischplatte da, sondern erfüllen mindestens eine zweite Funktion. Diese könnte in der Beherbergung von Technik bestehen, was durch die gitterartigen Bleche (der Kamera zugewandt) zusätzlich plausibel erscheint. Dann nämlich, wenn diese als Belüftungsgitter verstanden werden. Die Passung der vier Türme, Beine oder allgemein Eckelemente mit einem prototypischen Konzept von Tischbein ist fragwürdig, kann aber vermutlich nicht durch die spätere Interaktionsanalyse beantwortet werden. Eine Frage hingegen kann lauten, welche Teilnehmerkategorien i.S. von Funktionszuschreibungen es für die Eckelemente gibt.

\section{(4) Display}

Das Objekt wirkt in seiner Gänze so ausladend, dass von den Beobachtern vermutet wurde, die gesamte Umbauung des Displays habe mit dessen Funktion gar nichts zu tun. Das Display ist architektonisch das zentrale Element. Es erscheint optisch mittig und wird von einer deutlich abgesetzten Farbe gerahmt (rot). Annahmen zur Funktion der Farbgebung sind an dieser Stelle eher spekulativ: Die Farbe könnte das schwarze Display hervorheben, sie könnte aber auch eine Aufnahme des farbigen Schriftzugs am Boden sein. Insgesamt werden fünf verschiedene Farben verwendet, über deren Bedeutung bereits unter ,Sitzgelegenheiten' spekuliert wurde. Mit Blick auf den Bildausschnitt lässt sich zumindest sagen, dass die Verwendung von schwarz und rot genau entgegengesetzt auch im Hintergrund vorkommt, als Podeste unter den ausgestellten Autos. Im Vergleich dazu wirkt die Oberflächenfarbgebung des Tabletops viel stärker. Die Wirkung der Kontrastfarbe ist in dem Sinne ähnlich, als dass das Eigentliche hier das Display, dort das einzelne Auto - durch einen Rahmen hervorgehoben wird. Fragwürdig erschien einer Betrachterin die Verwendung sowohl, warmer als auch ,kalter' Farben. Die Farben könnten die Besucherbewegung steuern, also Nutzer anziehen. Was letztlich Zweck und Inhalt des Objekts ist, wird durch Anschauen aus mittlerer Distanz bzw. im Vorübergehen nicht ersichtlich. Wie in der Abbildung zu sehen, ist der größte Teil der Anzeige schwarz und nur in der Mitte sind farbige Rechtecke zu sehen. Die Beschriftungen sind so klein, dass man als Besucher gezwungen wird, näher heranzutreten, um die Anzeige lesen zu können. Auf der Abbildung ist die Aufschrift des Schildes nicht zu 
sehen, das auf der gegenüberliegenden Seite zu erkennen ist und durch das ein gedachtes Vorne konzeptualisiert wird.

Welchen ,Hinweisen' die Nutzer folgen, stellt sich als Frage für die Interaktionsanalyse. Dazu gehört auch die Frage der Bedientechnik, die in diesem Fall gleichzeitig eine sozialtopografische ist. $\mathrm{Zu}$ den ,unwritten rules des Museums gehört klassischerweise ein Berührungsverbot. Zudem legt ein Display dieser Größe zunächst eher eine Betrachtbarkeit als eine Berührbarkeit nahe. Für die Interaktionsanalyse ist also interessant zu verfolgen, ob es zwischen unterschiedlichen Nutzergruppen auch Unterschiede in der Aneignung des Displays gibt. Auf dem Bild ist nicht zu erkennen, welche Funktionalität das Display bereithält. Möglicherweise hilft die liegende Position bei der Entschlüsselung, ist diese doch für einen Monitor recht ungewöhnlich. Geradezu rätselhaft erscheinen die Aufsätze in den Ecken des Displays, die ebenso gut den Bein-Säulen zugerechnet werden könnten. Ob Nutzer sich damit beschäftigen, diese in ihre Aktionen aufnehmen oder sogar Verstehen signalisieren, bleibt in der Interaktionsanalyse zu beobachten.

\section{(5) Benutzung}

Die Frage, die sich vom Gesamteindruck über Farbgebung und Orientierung am Gerät zieht, lautet: Wozu ist dieses Objekt da? Die museale Rahmung lässt eine Vermittlung von Inhalten erahnen. Da es sich hier um ein Industriemuseum handelt, wird auch eine Thematisierung der Funktionalität erwartbar. Hier könnte die Interaktionsanalyse Aufschluss darüber geben, welche Theorien und Verstehensbekundungen zur Funktion des Tisches die Besucher einander mitteilen.

Die interaktionsarchitektonische Analyse zeigt, dass das Design des Tabletops viele Fragen offen lässt, die nur in der Interaktions(raum)analyse geklärt werden können:

- Wie wird das Objekt durch Besucher interpretiert bzw. konzeptualisiert?

- Wie lösen Nutzer das Problem der Aufenthaltsform am Display?

- Wie werden Deutungsprobleme in Teilnehmerkategorien offenbar?

- Gibt es nutzergruppenabhängige Arten der Nutzung des Geräts?

- Was wird durch die räumliche Begrenzung ermöglicht bzw. verhindert?

- Welche Teilnehmerkategorien i.S. von Funktionszuschreibungen gibt es für die Eckelemente?

- Welchen Hinweisen folgen die Nutzer?

- Welche Theorien und Verstehensbekundungen teilen die Benutzer einander mit? 
Allgemein steht über diesen im Detail herausgearbeiteten Fragen jene: Für welches interaktive Problem kann oder soll ein architektonisches Element eine Lösung anbieten? Die gemeinsame Betrachtung der räumlichen Gegebenheiten in der Datensitzung hat damit eine wichtige Grundlage für die weitere Gestaltung der Untersuchung gelegt. Mithilfe der herausgearbeiteten Fragen können Video-Aufzeichnungen von Interaktionsereignissen fokussiert analysiert werden. Im Hinblick auf eine weitere Datenerhebung können ebenfalls Rückschlüsse gezogen werden, die z.T. im weiteren Verlauf des Projekts bereits umgesetzt wurden. Diese sollen im folgenden Kapitel kurz skizziert werden.

\section{Rückschlüsse für das weitere Vorgehen}

Die in Kapitel 4 vorgestellte Analyse basiert auf Aufnahmen der ersten Nutzerstudien am CormforTable (Storz et al. 2015). Diese wurden vor allem mit Fokus auf den ethnografisch begleiteten Gestaltungsprozess angefertigt und waren für diesen Zweck völlig ausreichend. Eine Interaktionsarchitektur- und Interaktionsraumanalyse sieht sich jedoch vor gewisse Probleme gestellt. Die Perspektive der Kamera verzerrt das Bild, so dass Größen- und Abstandsverhältnisse nicht gut einschätzbar sind. Durch die Beschränkung auf eine ,Schussrichtung' ist die Annäherung von Besuchern an das Objekt nur für eine Seite nachvollziehbar, als Annährungsseite kommt aber auch die Gegenrichtung in Frage, die deshalb ebenfalls erfasst werden sollte. Für eine vollständige Erfassung des Ausstellungsraums erscheint es außerdem sinnvoll, Fotos von allen Seiten als Dokumente einzubringen, so dass auch die umgebende Architektur inklusive der anderen sichtbaren Exponate bzw. Geräte beschrieben werden kann. Problematisch an der gezeigten Aufnahme ist darüber hinaus die flächige Reflexion auf dem Display. Da dieses das Hauptelement des Tisches darstellt und rein flächenmäßig sehr präsent ist, dürfte die darauf zu sehende Grafik in der Analyse der Benutzbarkeitshinweise eine ganz erhebliche Rolle spielen. Und schließlich stellt die Ein-Perspektiven-Aufnahme die Interaktionsraumanalyse vor erhebliche Probleme. Die Sitzanordnung sorgt dafür, dass Teilnehmer (Benutzer) sich gegenseitig und das Display verdecken. In den Nutzungsstudien wird gar der Effekt nahegelegt, dass Besucher, die die Kamera sehen, sich so positionieren, dass sie möglichst nur von hinten zu sehen sind. Noch schwerwiegender ist die Nichtsichtbarkeit von Hand- und Armbewegungen, da Gesten und Bedienungstechniken für die Interaktionsraumanalyse eine entscheidende Rolle spielen. Für die geplante weitere Datenerhebung sind deshalb mindestens zwei Kameras vorgesehen. Dabei filmt eine die Totale, von der beide Zugangsseiten zum Tisch erfasst werden und alle Teilnehmer am Tisch zu sehen sind. Eine zweite filmt aus der Vogelperspektive das Geschehen am Tisch, wobei alle Hand- und Armgesten zweifelsfrei identifizierbar sind 
und wodurch die Sprecherzuordnung erheblich erleichtert werden dürfte (zur Verwendung der Vogelperspektive siehe auch Mondada (2007)). Eine dritte Kamera (als Gegenschuss) kann in Zweifelsfällen klarifizierende Aufnahmen liefern. Bereits die Ausstattung mit Totale und Vogelperspektive rückt jedoch relativ viel Aufnahmetechnik in den Wahrnehmungsbereich der Besucher, eine weitere Kamera dürfte diesen Effekt zweifelsohne noch verstärken. Mit einem insgesamt möglichst großen Ausschnitt des Raums soll der erwartbare Einfluss der Kameraführung (Mondada/Schmitt 2010:33) auf das Datum so klein wie möglich gehalten werden. Da im Zentrum der Analysen das Geschehen am Tisch steht, ist der Verlust eines größeren Bewegungsradius innerhalb der Ausstellung aus meiner Sicht vertretbar.

Die bisherigen Nutzungsstudien legen nahe, dass zur Bildung einer ersten Datenkollektion (vgl. z.B. Deppermann 2008:94ff.) besonders die Situationseröffnungen am Tabletop geeignet sind. Die Bildung einer Kollektion ist ein entscheidender Schritt, um fallübergreifende Analyseergebnisse zu erzielen. Dabei werden anhand von Kriterien (wie beispielsweise die Eröffnung einer Situation) mehrere Fälle zusammengestellt. Gegebenenfalls werden die Auswahlkriterien im Analyseprozess weiter modifiziert, bis sich ein konsistentes Muster ergibt (ebd.:95f.).

Als Situationseröffnung verstehe ich zunächst die Phase, in der sich mehrere Personen am Tisch zusammenfinden und auf das Display fokussieren. Die Beobachtung fokussierender Handlungen findet zunächst rein visuell statt und wird von den Teilnehmern beispielsweise durch Annäherung an den Tabletop und/oder Blickrichtung/-kontakt realisiert. Der Tisch wird in diesem Moment „signifikantes Objekt“ im Sinne eines ,gegenständlichen Mitkonstituenten von Interaktionsräumen, deren Zentrum und koordinativen Bezugspunkt [er] temporär bilde[t]“ (Mondada/Schmitt 2010:28). In der Analyse aller interaktiver Ressourcen räumen Mondada und Schmitt dieser Herangehensweise besondere Chancen ein, weil sich so eine eventuelle Überberücksichtigung der verbalen Äußerungen vermeiden lässt und neue Struktursegmente und Beschreibungskategorien möglich werden können (ebd.:30). Der Argumentation von Mondada und Schmitt folgend sind Situationseröffnungen im Rahmen von Interaktionsraumanalysen von zentraler Bedeutung. Denn „die Art und Weise, in der ein solcher Interaktionsraum als zentrale Voraussetzung für die intendierte zukünftige Interaktion gemeinsam hergestellt wird, ist dabei projektiv hinsichtlich zentraler thematischer, pragmatischer und sozialer Aspekte dieser zukünftigen Interaktion" (ebd.:39). Auf die hier vorgestellte Untersuchung bezogen heißt das, dass in der Situationseröffnung für den weiteren Verlauf des Spiels bzw. der Interaktion am Tabletop wichtige Weichen gestellt werden. Die Funktionslogik des Interfaces legt das ebenfalls nahe: Es muss sich eine Gruppe finden, die gemeinsam am Tabletop spielen will, die Funktion der Bedienung muss erschlossen und die Spielregeln müssen erlernt werden. Die Situationseröffnung endet zwangsläufig mit der Entscheidung: Abbruch - oder weitermachen. 


\section{Diskussion}

Wie in der Analyse in Kapitel 4 herausgearbeitet wurde, bleibt im Gesamteindruck des Tabletops vieles unklar. Seine Architektur erscheint nicht ausschließlich auf die Wirkung beim Besucher abzuzielen, sondern ebenso sehr auf die Lösung spezifischer Probleme. Daraus ergibt sich die Frage, welche Probleme durch die sichtbare Architektur gelöst werden. Diese Frage kann kaum durch die Interaktionsraumanalyse beantwortet werden. Antwort gibt hier letztlich der gut aufbereitete und reflektierte Design-Prozess, auf den an dieser Stelle lediglich verwiesen werden kann (z.B. Storz et al. 2015). Für den gesamten Erkenntnisprozess sollte sich diese dritte Datenquelle (neben Fotos und Videoaufnahmen) als äußerst wertvoll erweisen, ergibt sich doch eine Art Komplettierung von Benutzungshinweisen (Interaktionsarchitektur) und Benutzung (Interaktionsraum) durch die Intention oder Benutzungsabsicht (Designprozessanalyse).

Mit dem vorliegenden Artikel möchte ich zeigen, dass die interaktionslinguitisch orientierte Architekturanalyse für die Untersuchung von Mehr-Nutzer-Interaktion am ComforTable nutzbar ist. Es sollte auch deutlich werden, dass die Analyse von Interaktionsarchitektur und Interaktionsraum in einem konsequenten Nacheinander für die Untersuchung nutzerzentrierten Soft- und Hardwaredesigns überaus gewinnbringend ist. Eine detaillierte Betrachtung des Objekts im Raum und seiner tatsächlichen Benutzung lässt Rückschlüsse auf die Persuasionskraft des gestalteten Ausstellungsobjekts $\mathrm{zu}$ - in einer realen Umgebung. Sie bietet eine wichtige Annäherung an die Frage nach der ,sinnlich-intensiven Wirkung' des ComforTable und damit an die Frage, wie ein Exponat vom Ausstellungsobjekt zum (berührten) Spielobjekt wird.

\section{Literatur}

DEPPERMANN Arnulf, 2008, Gespräche analysieren, Wiesbaden.

FLICK Uwe, 2005, Standards, Kriterien, Strategien: zur Diskussion über Qualität qualitativer Sozialforschung, in: Zeitschrift für qualitative Bildungs- Beratungs- und Sozialforschung 6(2), S. $191-210$.

FriCKE Ellen, 2007, Origo, Geste und Raum: Lokaldeixis im Deutschen, Berlin [u.a.].

GÜLICH Elisabeth / MONDADA Lorenza, 2008, Konversationsanalyse: Eine Einführung am Beispiel des Französischen, Tübingen.

HAUSENDORF Heiko, 2012, Über Tische und Bänke, in: Hausendorf H./Mondada L./Schmitt R. (Hrsg.), Raum als interaktive Ressource, Tübingen, S. 136-189.

HAUSENDORF Heiko / KESSELHEIM Wolfgang, 2007, Die Multimodalität der Ausstellungskommunikation, in: Schmitt R. (Hrsg.), Koordination, Tübingen, S. 339-375.

HAUSENDORF Heiko / MONDADA Lorenza / SCHMITT Reinhold, 2012, Raum als interaktive Ressource, Tübingen.

HAUSENDORF Heiko / SCHMITT Reinhold, 2013, Interaktionsarchitektur und Sozialtopografie: Umrisse einer raumlinguistischen Programmatik, Arbeitspapiere des UFSP Sprache und Raum SpuR 01, Zürich. 
HAUSENDORF Heiko / SCHMITT Reinhold, 2014, Vier Stühle vor dem Altar, Arbeitspapiere des UFSP Sprache und Raum SpuR 03, Zürich.

KesselHEIM Wolfgang, 2012, Gemeinsam im Museum: Materielle Umwelt und interaktive Ordnung, in: Hausendorf H./Mondada L./Schmitt R. (Hrsg.), Raum als interaktive Ressource, Tübingen, S. $187-231$.

MondADA Lorenza, 2007, Interaktionsraum und Koordinierung, in: Schmitt R. (Hrsg.), Koordination, Tübingen, S. $55-128$.

MONDADA Lorenza / SCHMITT Reinhold, 2010, Zur Multimodalität von Situationseröffnungen, in: Mondada L./Schmitt R. (Hrsg.), Situationseröffnungen, Tübingen, S. 7-52.

MÜLleR-TOMFELdE Christian / FJELD Morten, 2010, Introduction: A Short History of Tabletop Research, Technologies, and Products, in: Müller-Tomfelde Ch. (Hrsg.), Building Interactive Multi-touch Surfaces, London, S. 1-24.

Norman Donald A., 1989, Dinge des Alltags (übers. von K. Cofer), Frankfurt a. M/New York.

Norman Donald A., 2004, Emotional Design: Why We Love (or Hate) Everyday Things, New York. PITSCH Karola, 2012, Museumsexponat, Alltagsobjekt oder Turngerät? - Zur Konstitution von Objekten im Interaktionsraum, in: Hausendorf H./ Mondada L./Schmitt R. (Hrsg.), Raum als interaktive Ressource, Tübingen, S. 233-273.

ScHMiтT Reinhold (Hrsg.), 2007, Koordination: Analysen zur multimodalen Interaktion, Tübingen. ScHMiTT Reinhold, 2013, Körperlich-räumliche Aspekte der Interaktion, Tübingen.

SCHroer Markus, 2006, Räume, Orte, Grenzen: Auf dem Weg zu einer Soziologie des Raums, Frankfurt a. M.

SCHWARZE Cordula, 2014, Theoretische und methodische Überlegungen zur Praxis der gesprächsanalytischen Datensitzung, in: Schwarze C./Konzett C. (Hrsg.), Interaktionsforschung - Gesprächsanalytische Fallstudien und Forschungspraxis, Berlin, S. 161-176.

Storz Michael / KANellopoulos Katja / FrAas Claudia / EIBL Maximilian, 2015, Designing with Ethnography: Tabletops and the Importance of their Physical Setup for Group Interactions in Exhibitions, in: i-com 14, S. 115-125.

STUKENBROCK Anja, 2015, Deixis in der face-to-face-Interaktion, Berlin/Boston.

StUTTERHEIM Christiane / KoHLMANN Ute, 2001, Beschreiben im Gespräch, in: Brinker K./Antos G./ Heinemann W./Sager S.F. (Hrsg.), Text- und Gesprächslinguistik (HSK Bd. 16.2), Berlin/New York, S. $1279-1292$.

Tuma René, 2013, Visuelles Wissen: Die Videoanalyse im Blick, in: Lucht P./Schmidt L.-M./ Tuma R. (Hrsg.), Visuelles Wissen und Bilder des Sozialen, Wiesbaden, S. 49-69.

Ueding Gert, 2008, Beredsamkeit der Formen - Anmerkung zu einer Rhetorik des Designs, in: Joost G./Scheuermann A. (Hrsg.), Design als Rhetorik, Basel, S. 81-88. 